\title{
Decomposição de resíduos culturais de cana-de-açúcar submetidos a diferentes doses de nitrogênio
}

\section{Decomposition of sugar cane crop residues under different nitrogen rates}

\author{
Douglas Costa Potrich ${ }^{1 *}$; Marlene Estevão Marchetti²; Diego Costa Potrich ${ }^{3}$; \\ Simone Cândido Ensinas ${ }^{1}$; Ademar Pereira Serra ${ }^{4}$; \\ Eulene Francisco da Silva ${ }^{5}$; Natália Hilgert de Souza ${ }^{6}$
}

\section{Resumo}

\begin{abstract}
A deposição de resíduos orgânicos através da colheita mecanizada da cana-de-açúcar é uma prática crescente no sistema de produção canavieiro. A manutenção desses resíduos na superfície do solo depende principalmente das condições ambientais. A adubação nitrogenada sobre os resíduos secos tendem a retardar a decomposição desses, proporcionando benefícios como o aumento da MOS. Diante disto, o objetivo da pesquisa foi avaliar o efeito da aplicação de diferentes doses de nitrogênio sobre os resíduos culturais de cana-de-açúcar, quanto a sua decomposição e contribuição para o sequestro de carbono no solo. O experimento foi realizado em Dourados-MS e consistiu de um delineamento experimental em blocos casualizados. Os resíduos secos foram colocados em litter bags e os tratamentos foram arranjados em parcelas subdivididas, sendo as quatro doses de nitrogênio $\left(0,50,100\right.$ e $\left.150 \mathrm{~kg} \mathrm{ha}^{-1} \mathrm{~N}\right)$ as parcelas, e as sete épocas de coleta $(0,30,60,90,120,150$ e 180 dias $)$ as subparcelas. Foram analisados a taxa de decomposição dos resíduos, carbono orgânico total e carbono lábil no solo. A aplicação de doses crescente de $\mathrm{N}$ proporcionou aumento nas suas taxas de decomposição. Apesar disso, destaca-se a aplicação de $\mathrm{N}$-mineral como uma estratégia de manejo para obter maiores teores de carbono lábil no solo.

Palavras-chave: Adubação nitrogenada, manutenção de palhada, carbono orgânico total, carbono lábil
\end{abstract}

\begin{abstract}
The deposition of organic residues through mechanical harvesting of cane sugar is a growing practice in sugarcane production system. The maintenance of these residues on the soil surface depends mainly on environmental conditions. Nitrogen fertilization on dry residues tend to retard decomposition of these, providing benefits such as increased SOM. Thus, the object of this research was to evaluate the effect of different doses of nitrogen on sugar cane crop residues, as its decomposition and contribution to carbon sequestration in soil. The experiment was conducted in Dourados-MS and consisted of a randomized complete block design. Dried residues were placed in litter bags and the treatments were arranged in a split plot, being the four nitrogen rates $\left(0,50,100\right.$ and $\left.150 \mathrm{~kg} \mathrm{ha}^{-1} \mathrm{~N}\right)$ the plots, and the seven sampling times $(0,30,60,90,120,150$ and 180$)$ the spit plots. Decomposition rates of residues, total organic carbon and labile carbon on soil were analysed. The application of increasing $\mathrm{N}$ doses resulted in an increase in their decomposition rates. Despite this, note also the mineral $\mathrm{N}$ application as a strategy to get higher levels of labile carbon in soil.
\end{abstract}

Key words: Nitrogen fertilization, residue conservation, total organic carbon, labile carbon

\footnotetext{
${ }^{1}$ Discentes de Doutorado em Agronomia, Programa de Pós Graduação em Agronomia, Universidade Federal da Grande Dourados, UFGD, Dourados, MS. E-mail: douglas_potrich@hotmail.com; simone_candido@hotmail.com

${ }^{2}$ Prof $^{a}$ Dra $^{\mathrm{a}}$, Universidade Federal da Grande Dourados, UFGD, Dourados, MS. E-mail: marlenemarchetti@ufgd.edu.br

${ }^{3}$ Eng $^{\circ}$ Agro $^{\circ}$, M.e em Agronomia, UFGD, Dourados, MS. E-mail: dcp_potrich@hotmail.com

${ }^{4}$ Analista A, Empresa Brasileira de Pesquisa Agropecuária, EMBRAPA-CNPGC, Campo Grande, MS. E-mail: ademar.serra@ embrapa.br

5 Prof ${ }^{\mathrm{a}} \mathrm{Dr}^{\mathrm{a}}$, Universidade Federal Rural do Semi-Árido, DCAT-UFERSA, Mossoró, RN. E-mail: eulenesilva@ufersa.edu.br

6 Prof $^{\mathrm{a}} \mathrm{Dr}^{\mathrm{a}}$, Universidade Estadual do Mato Grosso do Sul, UEMS, Dourados, MS. E-mail: natalia_hilgert@hotmail.com

* Autor para correspondência
} 


\section{Introdução}

A atividade antrópica tem elevado à concentração de gases do efeito estufa na atmosfera. Tais incrementos induziram a aumento da temperatura média terrestre e da taxa anual de elevação do nível do mar. No âmbito dessas mudanças climáticas globais, o solo e suas diferentes formas de uso e manejo estão em foco (COSTA et al., 2008), sobretudo porque o solo pode ser considerado como uma fonte ou sumidouro de $\mathrm{CO}_{2}$ atmosférico (CARVALHO et al., 2008).

O sistema de colheita da cana-de-açúcar com queimada elimina a matéria seca e aumenta a concentração de gás carbônico na atmosfera, contribuindo com o efeito estufa e diminuindo o teor de matéria orgânica no solo (SOUZA, et al., 2005). Já a colheita mecanizada, que está cada vez mais presente nos sistemas de produção no Brasil, contribui para o acúmulo de resíduos vegetais na superfície, mantendo o solo sempre coberto, promovendo o aumento nos seus estoques totais de carbono orgânico (COT) e nitrogênio total (NT) (PANOSSO et al., 2009).

Segundo Teixeira et al. (2010), a produção e manutenção de palhada sobre a superfície do solo constituem um dos principais fatores para o sucesso do sistema conservacionista de produção, sendo a mineralização dos resíduos dependente de fatores ambientais e químicos, que dependem, principalmente, da qualidade do material depositado (VITTI et al., 2008). Lal e Logan (1995) afirmaram que a taxa de decomposição nas regiões tropicais e subtropicais pode ser até 10 vezes mais acelerada do que em regiões temperadas.

Aqualidadedosmateriais podeser definida como a facilidade de serem utilizados pelos microrganismos do solo como fonte de energia e, ou, esqueletos de carbono para suas próprias estruturas (ROVIRA; VALEJO, 2007). Sendo assim, a predisposição de um resíduo à decomposição é dependente, principalmente, do teor inicial de lignina, nitrogênio (N) e das relações C/N (WANG; WANG; HUANG,
2008), lignina/N (VALENZUELA-SOLANO; CROHN, 2006).

A MOS é oriunda principalmente de resíduos orgânicos adicionados pelas plantas (exsudatos das raízes e restos culturais da senescência e manejo) que, através da fotossíntese, convertem $\mathrm{O} \mathrm{CO}_{2}$ atmosférico em compostos vegetais contendo carbono (BAYER; MIELNICZUK; MARTIN NETO, 2000). Quando ecossistemas nativos são alterados por atividades antrópicas, o equilíbrio dinâmico do $\mathrm{C}$ é quebrado e, normalmente, as entradas são menores do que as saídas, o que conduz à redução da quantidade e modificação da qualidade da MOS (CERRI; FEIGL; CERRI, 2008).

Observa-se que a adoção de um novo sistema de manejo do solo, promove mudanças rápidas nas frações orgânicas mais lábeis do solo, sendo essas consideradas apenas uma pequena fração do teor de $\mathrm{C}$ orgânico total e o indicador mais responsivo às alterações da qualidade do solo (STEVENSON, 1994). Por isso, além da importância de ordem agronômica, o aumento dos estoques de MOS é desejável devido ao potencial de armazenar $\mathrm{C}$ no solo em detrimento de uma diminuição da quantidade de $\mathrm{C}$ presente na atmosfera na forma de $\mathrm{CO}_{2}$ (LAL, 2004).

Diante disto, o objetivo da pesquisa foi avaliar o efeito da aplicação de diferentes doses de nitrogênio sobre os resíduos culturais de cana-de-açúcar, quanto a sua decomposição e contribuição para o sequestro de carbono no solo.

\section{Material e Métodos}

\section{Caracterização da área experimental}

O experimento foi realizado no campus da Universidade Federal da Grande Dourados UFGD, situada no município de Dourados-MS, latitude $22^{\circ} 11^{\prime} 58,8^{\prime}$ 'S, longitude $54^{\circ} 56^{\prime} 10,9$ 'O e altitude média de $457 \mathrm{~m}$, no período de 28 de abril de 2011 a 25 de outubro de 2011. As O clima, segundo a classificação de Köppen (1948), é do tipo 
Am (Tropical Monçônico), com precipitação média anual de $1455 \mathrm{~mm}$ e temperatura inferior a $18^{\circ} \mathrm{C}$ no mês mais frio e superior a $22^{\circ} \mathrm{C}$ no mês mais quente. Os dados de pluviosidade e de temperatura máximas e mínimas registrados durante o período da pesquisa estão apresentados no Tabela 1.

O solo foi classificado como Latossolo Vermelho distroférrico (EMBRAPA, 2009), com os seguintes valores médios dos atributos químicos e físicos: $\mathrm{pH}_{(\mathrm{CaCl} 2)}=4,37 ; \mathrm{Al}=0,7 \mathrm{cmol}_{\mathrm{c}} \mathrm{dm}^{-3} ; \mathrm{H}+\mathrm{Al}=1,6$ $\mathrm{cmol}_{\mathrm{c}} \mathrm{dm}^{-3} ; \mathrm{Ca}=5,1 \mathrm{cmol}_{\mathrm{c}} \mathrm{dm}^{-3} ; \mathrm{Mg}=2,3 \mathrm{cmol}_{\mathrm{c}} \mathrm{dm}^{-}$ 3; $\mathrm{P}=4,15 \mathrm{mg} \mathrm{dm}^{-3} ; \mathrm{K}=0,4 \mathrm{cmol}_{\mathrm{c}} \mathrm{dm}^{-3} ; \mathrm{Cu}=0,3 \mathrm{mg}$ $\mathrm{dm}^{-3} ; \mathrm{Zn}=0,9 \mathrm{mg} \mathrm{dm}{ }^{-3} ; \mathrm{Fe}=18,7 \mathrm{mg} \mathrm{dm}^{-3} ; \mathrm{Mn}=2,2$ $\mathrm{mg} \mathrm{dm}{ }^{-3}$; COT=18,8 $\mathrm{g} \mathrm{kg}^{-1} ; \mathrm{CL}=1,3 \mathrm{~g} \mathrm{~kg}^{-1}$; argila $=$ $630 \mathrm{~g} \mathrm{~kg}^{-1}$; areia $=100 \mathrm{~g} \mathrm{~kg}^{-1}$; silte $=270 \mathrm{~g} \mathrm{~kg}^{-1}$.

A área experimental foi cultivada em preparo convencional por nove anos na sucessão soja/ milho, sendo adotado o plantio direto nos últimos dez anos mantendo as mesmas culturas de verão e inverno, respectivamente. Todavia, por ocasião da implantação do experimento, a área foi mantida em pousio por 14 meses, desde fevereiro de 2010, para evitar interferência nos resultados pelo uso de insumos.

Tabela 1. Precipitação e média das temperaturas máxima e mínima para períodos decendiais no ano 2011.

\begin{tabular}{cccc}
\hline & Precipitação & T. Máx. & T. Mín. \\
\hline Decêndio & $(\mathrm{mm})$ & ${ }^{\circ} \mathrm{C}$ & ${ }^{\circ} \mathrm{C}$ \\
\hline 28/abril & 0,76 & 28,84 & 18,09 \\
08/maio & 10,92 & 24,86 & 13,60 \\
18/maio & 0,00 & 25,45 & 14,41 \\
28/maio & 0,00 & 26,16 & 14,12 \\
07/junho & 0,00 & 23,89 & 10,70 \\
17/junho & 9,39 & 24,14 & 10,91 \\
27/junho & 0,00 & 26,01 & 13,25 \\
07/julho & 11,18 & 17,20 & 8,38 \\
17/julho & 0,00 & 30,36 & 15,63 \\
27/julho & 2,29 & 26,61 & 14,67 \\
06/agosto & 1,27 & 23,58 & 11,36 \\
16/agosto & 0,00 & 33,36 & 18,82 \\
26/agosto & 0,25 & 25,14 & 11,79 \\
05/setembro & 0,00 & 32,05 & 15,94 \\
15/setembro & 1,27 & 28,88 & 13,69 \\
25/setembro & 1,52 & 29,32 & 16,43 \\
05/outubro & 8,00 & 32,87 & 17,20 \\
15/outubro & 40,60 & 30,24 & 21,19 \\
25/outubro & 25,30 & 26,20 & 19,00 \\
\hline
\end{tabular}

Fonte: Estação Meteorológica da UFGD. Dourados, MS (2011).

\section{Tratamentos e delineamento experimental}

O experimento consistiu de um delineamento experimental em blocos casualizado, com quatro repetições. Os tratamentos, doses de $\mathrm{N}$ e épocas de coleta, foram arranjados em parcelas subdivididas. Nas parcelas foram alocadas as quatro doses de $\mathrm{N}$ aplicadas sobre os resíduos orgânicos $(0,50,100$, $\left.150 \mathrm{~kg} \mathrm{ha}^{-1} \mathrm{~N}\right)$, utilizando como fonte a uréia $(45 \%$ de $\mathrm{N}$ ), e nas subparcelas as sete épocas de coleta dos materiais para analise $(0,30,60,90,120,150$ e 180 dias após aplicação dos tratamentos). 
Preparo do resíduo e implantação do experimento

O resíduo orgânico de cana-de-açúcar foi coletado em uma lavoura da Usina São Fernando, imediatamente após a colheita mecanizada da cana soca de segundo ano da variedade RB 93-5744. Esse resíduo foi levado para estufa de circulação ar forçado a $65^{\circ} \mathrm{C}$, até aferir peso constante. O material seco foi padronizado em tamanho médio de $1,5 \mathrm{~cm}$, com auxílio de tesoura, sendo que o mesmo era composto de folha, bainha, ponteiro e colmos.

Após a padronização do tamanho dos materiais secos, esses foram pesados e colocados dentro de sacos (litter bags). O litter bag é um saco de fios de nylon preto de malha $2 \mathrm{~mm}$ que foi cortado e costurado nas dimensões de 0,5 x 0,25 m para cobrir uma área de $0,125 \mathrm{~m}^{2}$ de solo, para avaliar a decomposição do resíduo. Foi então adicionado 25 g de resíduo orgânico de cana-de-açúcar em cada litter bag, o qual corresponde a uma aplicação de $2000 \mathrm{~kg} \mathrm{ha}^{-1}$ de material seco sobre o solo. A caracterização química do material seco utilizado no experimento está apresentado no Tabela 2 .

Tabela 2. Composição química do resíduo de cana-deaçúcar antes da aplicação dos tratamentos.

\begin{tabular}{cc}
\hline Composição Química & $\mathrm{g} \mathrm{kg}^{-1}$ \\
\hline Carbono & 707 \\
Nitrogênio & 24,1 \\
Relação C/N & 29,34 \\
Lignina & 221,1 \\
Lignina/N & 9,17 \\
Fósforo & 2,6 \\
Potássio & 17,0 \\
Cálcio & 8,0 \\
\hline
\end{tabular}

Fonte: Elaboração dos autores.

No dia 28 de abril de 2011 os litter bags foram depositados sobre o solo e na mesma data foi feita a adubação nitrogenada sobre os materiais. A aplicação de $\mathrm{N}$ foi realizada por meio de solução de uréia e água, preparada no Laboratório de Fertilidade do Solo da UFGD, à $0 ; 3,47 ; 6,94 ; 10,41 \%$ para as respectivas doses de $0,50,100$ e $150 \mathrm{Kg} \mathrm{ha}^{-1} \mathrm{~N}$.
Foi aplicado $40 \mathrm{~mL}$ dessa solução de uréia sobre os litter bags com o auxílio de copos aferidos em laboratório, onde foi preconizado uniformidade e constância no espalhamento do produto sobre toda $\mathrm{a}$ área do material seco.

Logo após a aplicação dos tratamentos, iniciouse a primeira coleta dos dados, referente ao tempo zero, sendo que a cada 30 dias se repetia a coleta, encerrando no dia 25 de outubro de 2011 (180 dias após aplicação dos tratamentos). A coleta mencionada refere-se à retirada dos litter bags do campo, e encaminhamento ao laboratório, e amostragem do solo abaixo da área de influencia dos materiais secos da cultura para determinação das variáveis.

\section{Variáveis}

Em laboratório, os resíduos remanescentes do interior dos litter bags eram retirados e limpos com o auxílio de pincel, colocados em sacos de papel e encaminhados a estufa de circulação forçada de ar à temperatura de $65^{\circ} \mathrm{C}$ até adquirirem peso constante. Após a pesagem de cada unidade experimental, foi possível calcular a matéria seca remanescente de acordo com a metodologia descrita por Olson (1963):

$$
\text { Matéria seca remanescente }=\frac{\text { MS x } 100}{\text { MSI }}
$$

onde: $\mathrm{MS}=$ matéria seca atual do resíduo; $\mathrm{MSI}=$ matéria seca inicial.

Para a determinação da taxa de decomposição, o modelo exponencial simples foi ajustado aos dados de matéria seca, de acordo com Olson (1963):

$$
\mathrm{X}=\mathrm{X}_{0} \mathrm{e}^{-k t}
$$

em que: $X$ é a quantidade de matéria seca remanescente após um período de tempo t, em dias; $\mathrm{X}_{0}$ é a quantidade de matéria seca inicial; e 
$k$ é a constante de decomposição. Reorganizando os termos dessa equação, foi possível calcular a constante de decomposição:

$$
k=\frac{\ln \left(\mathrm{X} / \mathrm{X}_{\underline{0}}\right)}{\mathrm{t}}
$$

De acordo com Thomas e Asakawa (1993), o tempo de meia-vida, $\mathrm{t}_{0,5}$, (tempo necessário para decomposição de $50 \%$ do resíduo inicial) e o $t_{0,05}$ (tempo necessário para que $95 \%$ dos resíduos se decomponham) foram obtidos por meio das equações:

$$
\mathrm{t}_{0,5}=\frac{\ln (2)}{k} \quad \mathrm{t}_{0,05}=\frac{3}{k}
$$

As amostras de solo foram coletadas com trado, tipo holandês, na profundidade 0-0,10 m. Essas foram acondicionadas em sacos plásticos, secas ao ar, destorroadas e passadas em peneira com malha de $2 \mathrm{~mm}$ (TFSA). As análises de carbono orgânico total e lábil no solo foram realizadas no Laboratório de Fertilidade do Solo da UFGD, de acordo com as metodologias descritas por Yeomans e Bremner (1988) e Loginow et al. (1987), respectivamente.

\section{Análise estatística}

Os dados de matéria seca remanescente foram submetidos à análise de variância e as equações matemáticas que melhor se ajustaram à decomposição da matéria seca foram obtidas por meio do software Sigma Plot 11.0.

Os teores de COT e CL no solo em função das doses de $\mathrm{N}$ aplicadas e das épocas de avaliação foram analisadas pelo teste $\mathrm{F}$ e, para o caso de diferenças significativas, foi realizada a análise de regressão linear múltipla utilizando-se o aplicativo computacional Saeg 9.1. A superfície de resposta foi ajustada para as doses de $\mathrm{N}$ aplicadas e épocas de avaliação nos casos em que a interação foi significativa $(\mathrm{p}<0,05)$, utilizando-se o Sigma Plot 11.0.

\section{Resultados e Discussão}

A decomposição da matéria seca de cana-deaçúcar foi influenciada pelas doses de $\mathrm{N}$ adicionadas, havendo interação significativa $(\mathrm{p}<0,01)$ na $k$ (Tabela 3). Foi observado no tratamento sem adição

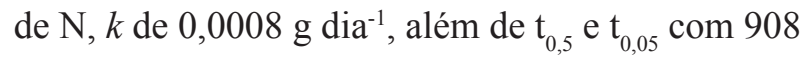
e 3931 dias, respectivamente. Quando foi aplicado $50 \mathrm{~kg} \mathrm{ha}^{-1}$ de $\mathrm{N}$ sobre a cana-de-açúcar, aumentou a $k$ para $0,0020 \mathrm{~g} \mathrm{dia}^{-1}$ e o tempo de meia vida desse passou para 339 dias, com diferença de 569 dias, tendendo a diminuir com o aumento da dose de N.

Tabela 3. Estimativas dos parâmetros da equação $X=X_{o} e^{-k t}$ ajustada aos dados de matéria seca remanescente de canade-açúcar quando submetidos às diferentes adubações nitrogenadas.

\begin{tabular}{cccccc}
\hline DOSE N & $\mathbf{X}$ & $\mathbf{R}^{\mathbf{2}}$ & $\boldsymbol{k}^{* *}$ & $\mathbf{t}_{0,5}$ & $\mathbf{t}_{0,05}$ \\
\hline $\mathrm{kg} \mathrm{ha}^{-1}$ & $\%$ & - & $\mathrm{g} \mathrm{dia}^{-1}$ & Dia & dia \\
$\mathbf{0}$ & 87,17 & 0,97 & 0,0008 & 908 & 3931 \\
$\mathbf{5 0}$ & 69,23 & 0,99 & 0,0020 & 339 & 1468 \\
$\mathbf{1 0 0}$ & 58,64 & 0,98 & 0,0030 & 234 & 1012 \\
$\mathbf{1 5 0}$ & 55,33 & 0,96 & 0,0033 & 211 & 912 \\
\hline
\end{tabular}

** Significativos a $1 \%$ pelo teste $\mathrm{t}$.

$\mathrm{X}=$ porcentagem de matéria seca remanescente aos 180 dias; $\mathrm{X}_{0}=$ porcentagem de matéria seca inicial $(100,00 \%) ; k=$ constante

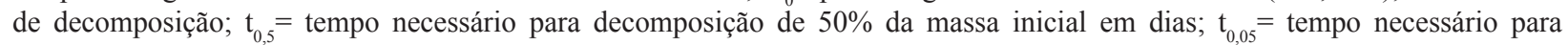
decomposição de $95 \%$ da massa inicial em dias.

Fonte: Elaboração dos autores. 
Aoaplicar $100 \mathrm{e} 150 \mathrm{kgha}^{-1}$ Nhouvedecomposição de 41,34 e $44,67 \%$ da massa seca inicial de canade-açúcar no período experimental (180 dias). Além disso, observa-se redução significativa no $t_{0,5}$ e $t_{0,05}$ da matéria seca de cana-de-açúcar na ordem de 697 e 3019 dias, respectivamente, quando comparado os tratamentos com ausência de $\mathrm{N}$ e aplicação de 150 $\mathrm{kg} \mathrm{ha}^{-1} \mathrm{~N}$. Isto mostra a importância da adubação nitrogenada sobre a palhada da cultura quando é necessário decomposição mais rápida do material vegetal presente sobre o solo.

Resultados semelhantes foram observados por Vitti et al. (2008) que ao analisar a mineralização da palhada de cana-de-açúcar submetida a diferentes doses de $\mathrm{N}$, observaram que na ausência da adubação nitrogenada houve menor decomposição do resíduo, restando após um ano agrícola $36 \%$ da massa seca inicial. Já ao aplicar 40, 80 e $120 \mathrm{~kg}$ $\mathrm{ha}^{-1}$ de $\mathrm{N}$ sobre os resíduos de cana-de-açúcar foi verificado maior decomposição da palhada quando comparados a ausência de adubação, porém não houve diferença significativa entre essas doses testadas, restando respectivamente 25,26 e $25 \%$ da massa seca remanescente.

Assis et al. (2003) também observaram incrementos na aceleração do processo de decomposição de resíduo de gramínea ao aplicar N. Para a cultura do sorgo as $k$ foram de 0,00509 e $0,00601 \mathrm{~g} \mathrm{dia}^{-1}$, para os tratamentos sem e com N $\left(30 \mathrm{~kg} \mathrm{ha}^{-1}\right)$. Essa diferença foi capaz de promover redução no $t_{0,5}$ em 31 dias, sendo o $t_{0,5}$ de 119 e 150 dias respectivos para presença e ausência de $\mathrm{N}$.

Segundo Aita (1997) isso ocorre porque a população e a atividade dos microrganismos decompositores são muito influenciados pela quantidade de $\mathrm{N}$ no solo, e o aumento na disponibilidade de $\mathrm{N}$ pode favorecer a taxa de decomposição. Além disso, aplicação de $\mathrm{N}$ diminui a relação $\mathrm{C} / \mathrm{N}$ da palhada de gramíneas, o que pode ativar o processo microbiano de decomposição, realizado principalmente por bactérias e fungos, que são considerados os decompositores primários (SMITH, 1994).

O teor de carbono orgânico total no solo, sob os materiais secos de cana-de-açúcar, apresentou diferença estatística $(\mathrm{p}<0,01)$ apenas para as épocas de avaliação, sendo ajustada regressão linear para este parâmetro. As doses de $\mathrm{N}$ aplicadas sobre os materiais de cana-de-açúcar não proporcionaram diferenças estatísticas ( $p>0,05)$. A equação linear ajustada para as épocas de avaliação mostraram que houve redução significativa no teor de COT no solo com o passar dos dias, sendo o maior teor observado no dia zero $\left(20,47 \mathrm{~g} \mathrm{~kg}^{-1}\right)$ e o menor aos 180 dias $\left(17,71 \mathrm{~g} \mathrm{~kg}^{-1}\right)$ (Figura 1).

Os resíduos da cana-de-açúcar possui elevado teor de lignina, o qual indica principalmente folhas duras e de difícil decomposição. As doses de $\mathrm{N}$ aplicadas provavelmente não influenciaram nem na população de microrganismos decompositores, já que foi verificado o efeito "priming" em todas as doses testadas, ou seja, ocorreu a mineralização do $\mathrm{C}$ existente no solo. Sendo assim, o solo foi perdendo $\mathrm{C}$ à medida que se passou os dias, já que a entrada de $\mathrm{C}$ pelos resíduos de cana-de-açúcar não foram suficientes para suprir as saídas de $\mathrm{C}$ no solo, via $\mathrm{CO}_{2}$.

Zanatta et al. (2007) evidenciaram que para manter o estoque inicial de $\mathrm{C}$ no solo, deve-se principalmente adequar a quantidade de resíduo a ser depositado sobre o solo, uma vez que isso tende a variar de acordo com o sistema de manejo do solo e das adubações nitrogenadas. Além disso, temse observado que dentro de um clima particular, a concentração química inicial é considerada o melhor preditor da taxa de decomposição de um resíduo, especialmente nas regiões dos trópicos. 
Figura 1. Superfície de resposta para o teor de carbono orgânico total no solo em função das épocas de avaliação dentro de cada dose de nitrogênio aplicada sobre a matéria seca de cana-de-açúcar. Dourados-MS, 2011.

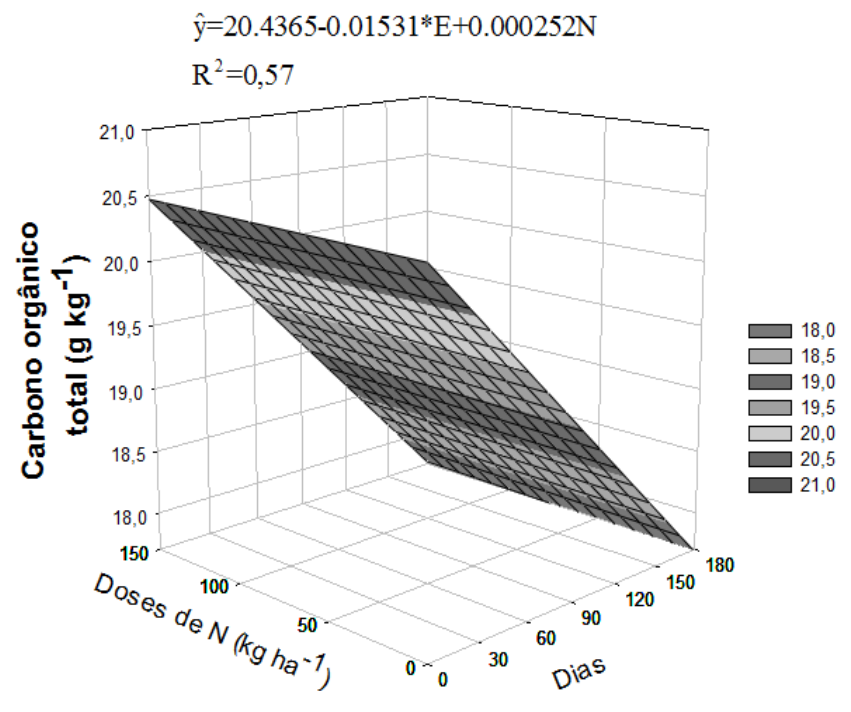

Fonte: Elaboração dos autores.

Ao avaliar o teor de carbono lábil no solo sob os resíduos de cana-de-açúcar pode-se registrar que houve interação significativa $(\mathrm{p}<0,01)$ entre as doses de $\mathrm{N}$ e as épocas de avaliação. Verificouse ajuste linear crescente do teor de CL no solo, em função das doses de $\mathrm{N}$ aplicadas. Já as épocas de amostragem proporcionaram ajuste quadrático, sendo observado o ponto máximo de CL no solo $\left(2,49 \mathrm{~g} \mathrm{~kg}^{-1}\right)$ aos 124 dias no tratamento com aplicação de $150 \mathrm{~kg} \mathrm{ha}^{-1} \mathrm{~N}$ (Figura 2).

A deposição de resíduos culturais de canade-açúcar na superfície do Latossolo em pousio mostrou-se uma prática eficiente para aumentar o teor de CL no solo, principalmente quando aliada a adubação nitrogenada. $\mathrm{O}$ comportamento do $\mathrm{C}$ no solo aconteceu em função da disponibilidade inicial de materiais prontamente disponíveis que foram decompostos e assim elevaram o teor de CL no solo. Ao final das épocas de avaliação foi verificada redução nos teores, sendo este fato atribuído a maior recalcitrância dos resíduos remanescentes. Segundo Lucas (2004), os teores de CL no solo podem declinar rapidamente, porém sua recuperação também pode ser rápida, o que sugere o uso do CL como um indicador sensível da dinâmica do $\mathrm{C}$ no sistema. 
Figura 2. Superfície de resposta para o teor de carbono lábil no solo em função das épocas de avaliação dentro de cada dose de nitrogênio aplicada sobre a matéria seca de cana-de-açúcar. Dourados-MS, 2011.

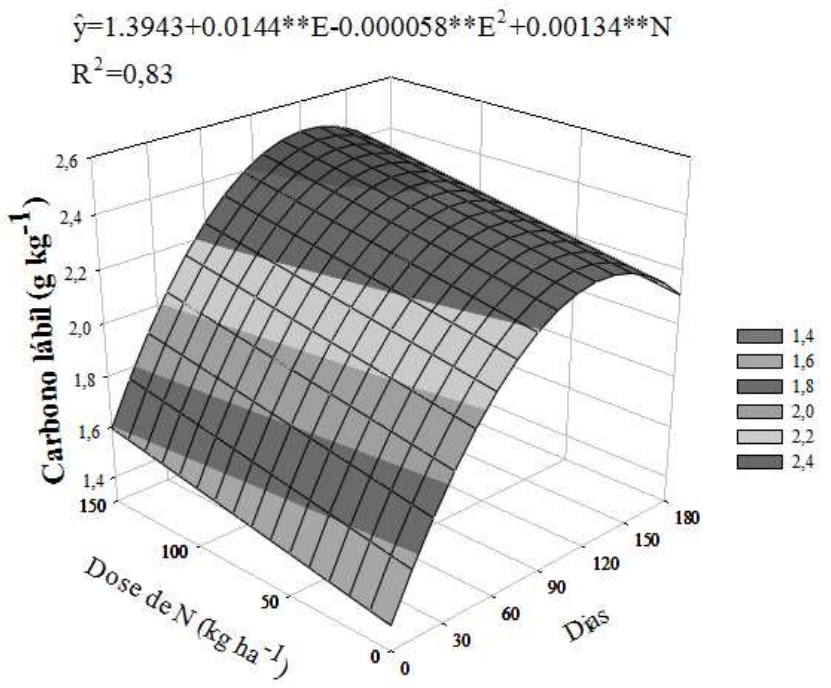

Fonte: Elaboração dos autores.

\section{Conclusões}

A adubação nitrogenada sobre resíduos secos de cana-de-açúcar aumenta a taxa de decomposição à medida que se aumenta a dose de $\mathrm{N}$ aplicada, nas condições edafoclimáticas de Dourados-MS.

A deposição de $2000 \mathrm{~kg} \mathrm{ha}^{-1}$ de resíduo de canade-açúcar é insuficiente para aumentar o teor de carbono orgânico total no solo, porém, é um fator essencial para o incremento de carbono lábil no solo ao longo do tempo. Além disso, destaca-se a aplicação de N-mineral como uma estratégia de manejo para obter maiores teores.

\section{Agradecimentos}

Os autores agradecem ao Conselho Nacional de Desenvolvimento Científico e Tecnológico (CNPq) pelo bolsa concedida ao primeiro autor.

\section{Referências}

AITA, C. Dinâmica do nitrogênio no solo durante a decomposição de plantas de cobertura: efeito sobre a disponibilidade de nitrogênio para a cultura de sucessão. In: FRIES, M. R.; DALMOLIN, R. S. D. (Ed.). Atualização em recomendação de adubação e calagem: ênfase em plantio direto. Santa Maria: RFSM;_Palltti, 1997, p. 76-111.

ASSIS, E. P. M.; CORDEIRO, M. A. S.; PAULINO, H. B.; CARNEIRO, M. A. C. Efeito da aplicação de nitrogênio na atividade microbiana e na decomposição da palhada de sorgo em solo de cerrado sob plantio direto. Pesquisa Agropecuária Tropical, Goiás, v. 33, n. 2, p. 107-112, dez. 2003.

BAYER, C.; MIELNICZUK, J.; MARTIN NETO, L. Efeito de sistemas de preparo e de cultura na dinâmica da matéria orgânica e na mitigação das emissões de $\mathrm{CO}_{2}$. Revista Brasileira de Ciência do Solo, Viçosa, MG, v. 24, n. 3, p. 599-607, jun. 2000.

CARVAlHO, J. L. N.; CERRI, C. E. P.; FEIGL, B. J.; PICOLlO, M. C.; GODINHO, V. P.; CERRI, C. C. Carbon sequestration in agricultural soils in the Cerrado region of the Brazilian Amazon. Soil and Tillage Research, Amsterdam, v. 103, n. 2, p. 342-349, 2008. 
CERRI, C. E. P.; FEIGL, B.; CERRI, C. C. Dinâmica da matéria orgânica do solo na Amazônia. In: SANTOS, G. de A.; SILVA, L. S. da; CANELLAS, L. P.; CAMARGO, F. de O. (Ed.). Fundamentos da matéria orgânica do solo: ecossistemas tropicais e subtropicais. 2. ed. Porto Alegre: Metrópole, 2008. p. 325-358.

COSTA, F. de S.; BAYER, C.; ZANATTA, J. A.; MIELNICZUK, J. Estoque de carbono orgânico no solo e emissões de dióxido de carbono influenciadas por sistemas de manejo no sul do Brasil. Revista Brasileira de Ciência do Solo, Viçosa, MG, v. 32, n. 1, p. 323-332, 2008.

EMPRESA BRASILEIRA DE PESQUISA AGROPECUÁRIA - EMBRAPA. Sistema brasileiro de classificação de solos. Rio de Janeiro: Centro Nacional de Pesquisa de Solos/Embrapa Solos, 2009. 412 p.

ESTAÇÃO METEOROLÓGICA DA UFGD. Dourados, MS: [s.n], 2011. Disponível em: <http://www.ufgd.edu. br/clima >. Acesso em: 22 out. 2013.

KÖPPEN, W. Climatologia: con un estudio de los climas de la tierra. México: Fondo de Cultura Económica, 1948. $478 \mathrm{p}$.

LAL, R. Soil carbon sequestration to mitigate climate change. Review Geoderma, Amsterdam, v. 123, n. 1-2, p. 1-22, 2004.

LAL, R.; LOGAN, T. J. Agricultural activities and greenhouse gas emissions from soils of the tropics. In: LAL, R.; KIMBLE, J. M.; LEVINE, E.; STEWART, B. A. (Ed.). Soil management greenhouse effect. Boca Raton: CRC Press, 1995. p. 293-307.

LOGINOW, W.; WISNIEWSKI, W.; GONET, S. S.; CIESCINSKA, B. Fractionation of organic carbon based on susceptibility to oxidation. Polish Journal of Soil Science, Poland, v. 20, n. 1, p. 47-52, 1987.

LUCAS, S. T. Evaluation of labile soil carbon test for prediction of soil productivity response to organic matter management. 2004. Dissertação (B.S. in Agricultural Sciences and Technology) - Department of Natural Resource Sciences and Landscape Architecture. University of Maryland, College Park.

OLSON, J. S. Energy storage and the balance of producers and decomposers in ecological systems. Ecology, Tempe, v. 44, n. 2, p. 322-331, 1963.

PANOSSO, A. R.; MARQUES JÚNIOR, J.; PEREIRA, G. T.; SCALA JÚNIOR, N. Spatial and temporal variability of soil $\mathrm{CO}_{2}$ emission in a sugarcane area under green and slash and-burn managements. Soil and Tillage Research, Amsterdam, v. 105, n. 2, p. 275-282, 2009.

ROVIRA, P.; VALLEJO, R. V. Labile, recalcitrant, and inert organic matter in Mediterranean forest soils. Soil
Biology and Biochemistry, Oxford, v. 39, p. 202-215, 2007. Especial.

SMITH, J. L. Cycling of nitrogen through microbial activity. In: HATFIELD, J. L.; STEWART, B. A. (Ed.). Soil biology: effects on soil quality. Boca Raton: CRCPress, 1994. p. 91-120.

SOUZA, Z. M.; PRADO, R. M.; PAIXÃO, A. C. S.; CESARIN, L. G. Sistemas de colheita e manejo da palhada de cana-de-açúcar. Pesquisa Agropecuária Brasileira, Brasília, v. 40, n. 3, p. 271-278, 2005.

STEVENSON, F. J. Humus chemistry: genesis, composition, reactions. 2. ed. New York: J. Wiley, 1994. $496 \mathrm{p}$.

TEIXEIRA, C. M.; CARVALHO, G. J. de; SILVA, C. A.; ANDRADE, M. J. B. de; PEREIRA, J. M. Liberação de macronutrientes das palhadas de milheto solteiro e consorciado com feijão-de-porco sob cultivo de feijão. Revista Brasileira de Ciência do Solo, Viçosa, MG, v. 34, n. 2, p. 497-505, 2010.

THOMAS, R. J.; ASAKAWA, N. M. Decomposition of leaf litter from tropical forage grasses and legumes. Soil Biology and Biochemistry, Oxford, v. 25, n. 10, p. 13511361, 1993.

VALENZUELA-SOLANO, C.; CROHN, D. M. Are decomposition and $\mathrm{N}$ release from organic mulches determined mainly by their chemical composition? Soil Biology and Biochemistry, Oxford, v. 38, n. 2, p. $377-$ 384, 2006.

VITTI, A. C.; TRIVELIN, P. C. O.; CANTARELLA, H.; FRANCO, H. C. J.; FARONI, C. E.; OTTO, R.; TRIVELIN, M. O.; TOVAJAR, J. G. Mineralização da palhada e crescimento de raízes de cana-de-açúcar relacionados com a adubação nitrogenada de plantio. Revista Brasileira de Ciência do Solo, Viçosa, MG, v. 32, p. 2757-2762, 2008. Especial.

WANG, Q.; WANG, S.; HUANG, Y. Comparisons of litterfall, litter decomposition and nutrient return in a monoculture Cunninghamia lanceolata and a mixed stand in southern China. Forest Ecology Management, Shenyang, v. 225, n. 3-4, p. 1210-1218, 2008.

YEOMANS, J. C.; BREMNER, J. M. A rapid and precise method for routine determination of organic carbon in soil. Communications in Soil Science and Plant Analysis, New York, v. 19, n. 13, p. 1467-1476, 1988.

ZANATTA, J. A.; BAYER, C.; DIECKOW, J.; VIEIRA, F. C. B.; MIELNICZUK, J. Soil organic carbon accumulation and carbon costs related to tillage, cropping systems and nitrogen fertilization in a subtropical Acrisol. Soil and Tillage Research, Amsterdam, v. 94, n. 2, p. 510519, 2007. 
